\title{
Crustáceos ectoparásitos y epibiontes de ballenas jorobadas, Megaptera novaeangliae (Cetacea: Balaenopteridae) en el Pacífico colombiano
}

\author{
Isabel Cristina Avila Jiménez ${ }^{1}$, Luis Miguel Cuellar Reina ${ }^{2}$ y Jaime Ricardo Cantera Kintz ${ }^{2}$ \\ ${ }^{1}$ Grupo de Ecología Animal. Departamento de Biología, Universidad del Valle. Calle 13 No.100-00, Cali, Colombia; isabel_c_avila@yahoo.com \\ ${ }^{2}$ ECOMANGLARES. Grupo de Ecología de Estuarios y Manglares. Departamento de Biología, Universidad del Valle. Calle 13, No. 100-00, \\ Cali, Colombia; luismbios@yahoo.es; jaime.cantera@correounivalle.edu.co
}

Recibido 3-III-2011 Corregido 25-V-2011 Aceptado 10-VI-2011

\begin{abstract}
Crustaceans ectoparasites and epibionts of humpback whales, Megaptera novaeangliae (Cetacea: Balaenopteridae), in the Colombian Pacific. We recorded three species of crustacean ectoparasites and epibionts in two calves and four adults of humpback whale (Megaptera novaeangliae) that in the breeding season 2010 visited PNN Gorgona and Bahía Málaga, Colombian Pacific: the amphipod Cyamus boopis and the barnacles Coronula diadema and Conchoderma auritum. These crustaceans adhere to calves and adults and are present in the caudal fin, dorsal fin, back of the body (near the blowhole) and ventral side of the lower jaw. Ectoparasites and epibionts recorded here may influence the movement, feeding and behavior of whales. We found a calf of no more than three months of age with epibionts: epibionts seem to attach to whales in tropical waters, most likely in Colombian waters.
\end{abstract}

\section{KEY WORDS}

Crustacean, amphipods, barnacles, Megaptera novaeangliae, Colombian Pacific.

\section{RESUMEN}

Se registran tres especies de crustáceos ectoparásitos y epibiontes en dos ballenatos y cuatro adultos de ballena jorobada (Megaptera novaeangliae) que visitaron el PNN Gorgona y Bahía Málaga en el Pacífico colombiano, en la temporada reproductiva del año 2010: el anfípodo Cyamus boopis y los cirripedios Coronula diadema y Conchoderma auritum. Se encontró que estos crustáceos se establecen en las aletas caudal y dorsal, en la parte dorsal del cuerpo cerca al espiráculo, y en la parte ventral de la mandíbula inferior. Se adhieren tanto a ballenas adultas como a ballenatos de corta edad. Los ectoparásitos y los epibiontes registrados pueden incidir en el desplazamiento de las ballenas, en la alimentación y en su comportamiento. Los epibiontes se adhieren a las ballenas en aguas tropicales, muy posiblemente en aguas colombianas, pues encontramos un ballenato de a lo sumo tres meses de edad con epibiontes.

\section{PALABRAS CLAVE}

Crustáceos, anfípodos, balanos, ballenas, Megaptera novaeangliae, Pacífico colombiano.
La ballena jorobada, Megaptera novaeangliae, es una especie migratoria, cosmopolita, costera, que se alimenta en las altas latitudes y se reproduce en zonas tropicales y subtropicales (Dawbin 1966). Esta especie visita anualmente entre junio y noviembre el Pacífico colombiano para reproducirse y criar, después de una migración de $8500 \mathrm{~km}$ desde la península Antártica y el estrecho de Magallanes en Chile (Stevick et al. 2004, Acevedo et al. 2007). La ballena jorobada es una especie principalmente costera y su supervivencia se encuentra afectada por factores antropogénicos, tales como la colisión con embarcaciones, la degradación del hábitat, la contaminación biológica (e.g. aguas de lastre descargadas por los barcos) y química (e.g. derrame de combustible), el acoso y la persecución por las embarcaciones de observación turística de ballenas, el enmallamiento accidental en redes de pesca y las perturbaciones y daños causados por el ruido (Corkeron 1995, Capella et al. 2001, Laist et al. 2001, Van Waerebeek et al. 2007, Van Bressem et al. 2009).

Por otro lado, las ballenas jorobadas son presas naturales de la orca (Orcinus orca) (Whitehead \& Glass 1985, D'Vincent et al. 1989, Flórez-González et al. 1994, Weller 2002) y ocasionalmente de la falsa orca (Pseudorca crassidens) y 
de algunas especies de tiburones, como el tiburón tigre (Galeocerdo cuvier) (Weller 2002). Además son hospederas de diversos parásitos, endo y ectoparásitos, y sirven de sustrato para algunos organismos epibiontes que se les adhieren a su piel. Entre los endoparásitos encontrados, el nematodo del género Crassicauda representa el más grave problema para las jorobadas y otros balenopteridos; sin embargo, se desconoce la tasa de mortalidad ocasionada por ellos (Lambertsen 1986, Raga et al. 2009). Los ectoparásitos más comunes son los ciámidos (Amphipoda), pequeños crustáceos que se alimentan de la piel de la ballena y que se establecen en lugares protegidos de la piel de la ballena o en estructuras que les permiten mantenerse unidos a ella. No obstante, al parecer es poco probable que el daño que causan a la salud y al bienestar de las ballenas sea significativo, pero puede representar laceraciones que después causen vulnerabilidad a otros problemas de salud (Raga et al. 2009). Los epibiontes más comunes en las ballenas jorobadas son los crustáceos denominados "balanos" (Cirripedia) del género Coronula, los cuales utilizan a la ballena como sustrato para instalarse y alimentarse del plancton, generalmente los ciámidos se encuentran cerca de la base de los balanos (Clarke 1966, Rowntree 1996, Fertl \& Newman 2009).

Algunos autores sugieren que los balanos pueden crecer en partes del cuerpo que afectan el libre desempeño y el desplazamiento de los cetáceos (Clarke 1966, Rowntree 1996, Fertl \& Newman 2009). La alta presencia de balanos aumenta además el peso que las ballenas deben mover: Slijper (1962) registró un individuo de ballena jorobada llevando $450 \mathrm{~kg}$ de balanos en su cuerpo. Adicionalmente, se han registrado algunos casos cuya infestación por especímenes de Coronula afectó gravemente la maxila y la mandíbula (deformaciones, fracturas y desviaciones de los huesos) de un delfín moteado (Stenella attenuata graffmani) y de un cachalote (Physeter macrocephalus) (Clarke 1966, Perrin 1969). En contraste con lo anterior, algunos autores han sugerido que los balanos son simbiontes de las ballenas jorobadas: la presencia de Coronula en las aletas dorsal y caudal de las ballenas podría ser usada como un arma para defenderse del ataque de orcas, pues incrementa la posibilidad de causar heridas en ellas (Ford \& Reeves 2008), y podría beneficiar a los machos al ser usada como un arma en la competencia intrasexual (Pierotti et al. 1985). Dyer (1989) sugiere que el establecimiento de Coronula diadema podría ayudar a la ballena jorobada a ecolocalizar sus presas debido a las propiedades acústicas que podría tener su exoesqueleto en forma de hexágono; cuando $C$. diadema se alimenta de fitoplancton emite chasquidos que pueden ser amplificados y transmitidos por su exoesqueleto y este sonido puede ser usado por la ballena para ecolocalizar sus presas.
Aunque la presencia de ectoparásitos y epibiontes es común en ballenas jorobadas y parece no representar una amenaza para la supervivencia de la especie, sí podrían afectar el desempeño normal de las ballenas (Clapham 2000, Fertl \& Newman 2009, Raga et al. 2009). Por otra parte, diversos autores afirman que el estudio de los parásitos asociados a las ballenas es una herramienta esencial para comprender mejor la dinámica poblacional de las ballenas en un área, ya que pueden ser usados como un marcador biológico que indica las rutas de migración y alimentación, así como su estado general de salud (Balbuena et al. 1995, Mackenzie 2002). Además, el estudio de la distribución geográfica de los epibiontes, particularmente los registros fósiles de Coronula, puede proveer información suplementaria y valiosa para los estudios paleobiogeográficos y paleoecológicos acerca de las rutas de migración de las ballenas en el pasado (Bianucci et al. 2006). A pesar de la importancia de los estudios de la fauna ectoparásita y de los epibiontes asociados a las ballenas jorobadas, en Colombia este tema es poco conocido. El presente trabajo registra la presencia de ectoparásitos y epibiontes en ballenas jorobadas en el Pacífico colombiano.

\section{METODOLOGÍA}

Se analizaron organismos ectoparásitos y epibiontes recolectados de un ballenato que colisionó con una embarcación de motor fuera de borda el 26 de julio de 2010 en el Parque Nacional Natural Gorgona (Cauca, Colombia), a $500 \mathrm{~m}$ de Playa Blanca $\left(2^{\circ} 56^{\prime} 39^{\prime \prime} \mathrm{N}\right.$ y $\left.78^{\circ} 11^{\prime} 14^{\prime \prime} \mathrm{W}\right)$. La colisión causó golpes en el ballenato y el desprendimiento de un pedazo de piel, que quedó adherido a la embarcación con ectoparásitos y epibiontes de este animal. Los organismos recolectados fueron identificados en el laboratorio utilizando un estereoscopio Motic 1.0X-4.0X, fueron fotografiados con una cámara Panasonic Lumix DMC-LZ5, conservados en etanol al 70\%, y posteriormente incluidos en la colección biológica del PNN Gorgona. Por otro lado, también se analizaron fotografías de cinco individuos (un ballenato y cuatro adultos) de ballenas jorobadas con ectoparásitos y epibiontes que visitaron Bahía Málaga, Valle, Colombia $\left(3^{\circ} 55^{\prime} 23^{\prime \prime} \mathrm{N}\right.$ y $77^{\circ} 20^{\prime} 21^{\prime \prime} \mathrm{W}$ ) en agosto de 2010. Los organismos ectoparásitos y epibiontes fueron analizados y fotografiados utilizando una cámara Nikon D50 con lente $55-200 \mathrm{~mm}$. Para la identificación de las especies ectoparásitas y epibiontes se utilizaron los trabajos de Yuk-Maan (1967), Cintron-De Jesus (2001), Holthuis \& Fransen (2004) y Bianucci et al. (2006). 


\section{RESULTADOS}

En el ballenato del PNN Gorgona se identificó el ectoparásito Cyamus boopis y los balanos Coronula diadema y Conchoderma auritum. Respecto al C. boopis, se encontraron nueve especímenes machos en la base del caparazón de C. diadema. Las dimensiones de C. boopis oscilaron entre 3,5 y $7.9 \mathrm{~mm}$ de longitud (desde la probosis hasta el pleón) y de 1-3,75mm de anchura. Los individuos encontrados poseen branquias simples y filiformes en los segmentos tres y cuatro, con sólo un par de branquias accesorias lobuladas (en la base de cada branquia), superficie dorsal lisa, cuerpo de color blanco hueso, lados del pereón no paralelos, con solo un par de espinas en los segmentos cinco, seis y siete (ventral) (Fig. 1).
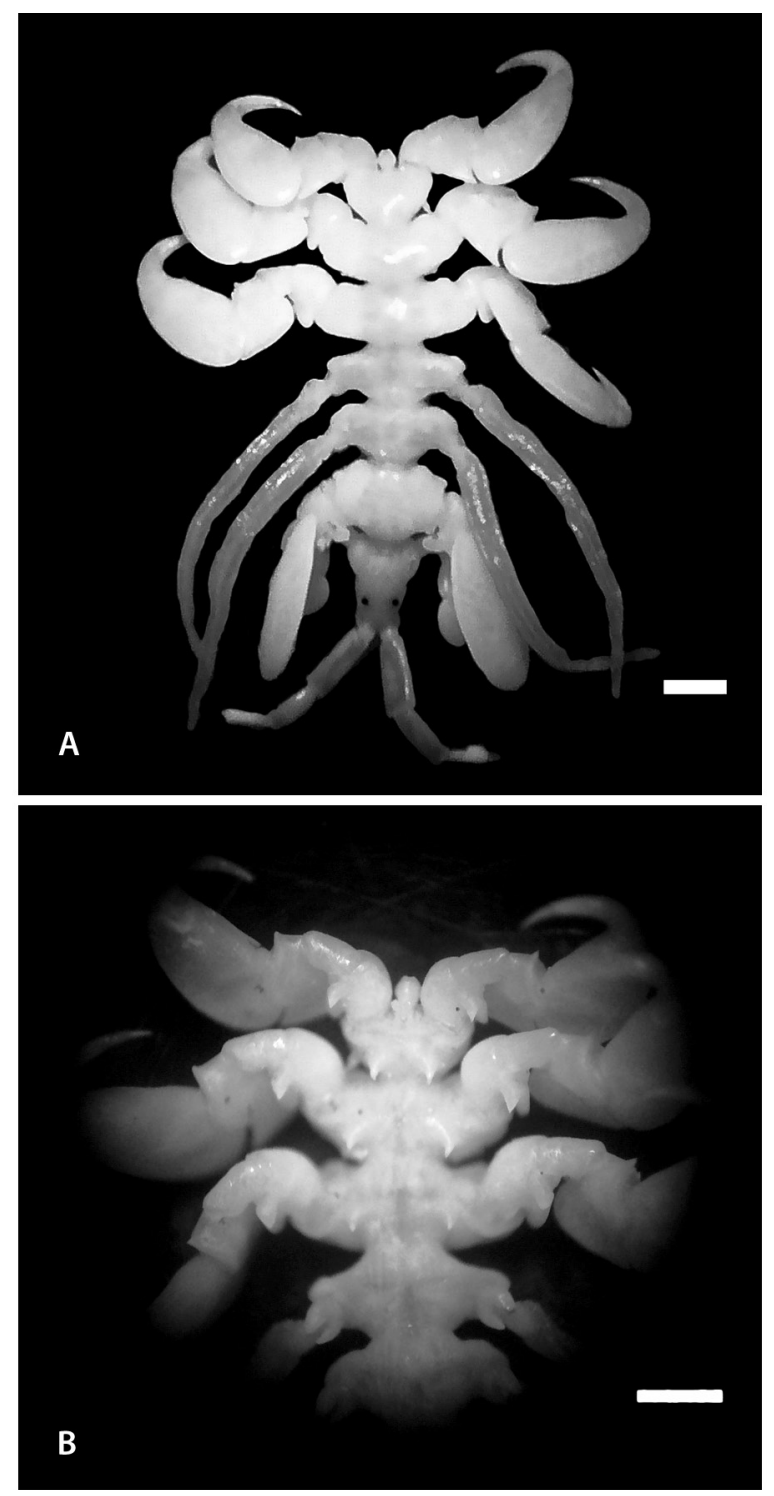

Se registraron dos C. diadema en el ballenato del PNN Gorgona, uno de ellos completamente destruido, el otro en buen estado. El especímen en buen estado posee un caparazón con corona alta, opérculo hexagonal y más grande que su base, radios altos y amplios con bordes crenados, tergo vestigial o ausente, costillas amplias y altas, y la base de la concha es muy profunda (cóncava). Tiene un diámetro basal de $49,4 \mathrm{~mm}$ y un altura desde la base hasta el opérculo de $29,4 \mathrm{~mm}$ (Fig. 2 y $3 \mathrm{~A}$ ); en su base se encontraron restos de piel del ballenato y adheridos a él habían dos especímenes de C. auritum (Fig. 3B).

Se recolectaron 26 individuos de $C$. auritum en el ballenato del PNN Gorgona, con longitudes axiales que oscilaron entre 4,4 y 41,5mm (desde la base del pedúnculo hasta el final de la abertura del capitulum). Estos individuos son pedunculados sin placas calcáreas, de consistencia densa y compresible, de textura lisa, coloración purpura oscuro a marrón; capitulum moteado con dos valvas reducidas, separadas entre sí, membrana gruesa (parecida a una oreja), valvas bien desarrolladas (aun en juveniles), escudo pequeño, bilobulado y ligeramente calcificado, tergo pequeño en adultos, totalmente desarrollado, pedúnculo cilíndrico sin placas, y claramente delimitado por una sutura justo antes del capitulum (Fig. 4).

De los animales fotografiados en Bahía Málaga durante la temporada 2010, se registraron cuatro adultos y un ballenato con epibiontes, los cuales fueron identificados como Coronula diadema y Conchoderma auritum. La presencia de $C$. diadema y $C$. auritum fue registrada en tres adultos de ballena jorobada en los bordes de la aleta caudal, en el dorso y cerca al espiráculo (Fig. 5, 6 y 7). Se registró sólo C. diadema en la aleta dorsal de un adulto (Fig. 8) y en la parte ventral de la mandíbula inferior de un ballenato nacido en la temporada 2010, con una edad estimada de tres meses (aproximadamente $6 \mathrm{~m}$ de longitud y parches de coloración blanca; Fig. 9).

FIG. 1. (A) Macho de Cyamus boopis recolectado de un ballenato de ballena jorobada en el PNN Gorgona; (B) Detalle ventral de espinas. (Escala: $1 \mathrm{~mm}$ ).

Fotos: Luis Miguel Cuellar. 


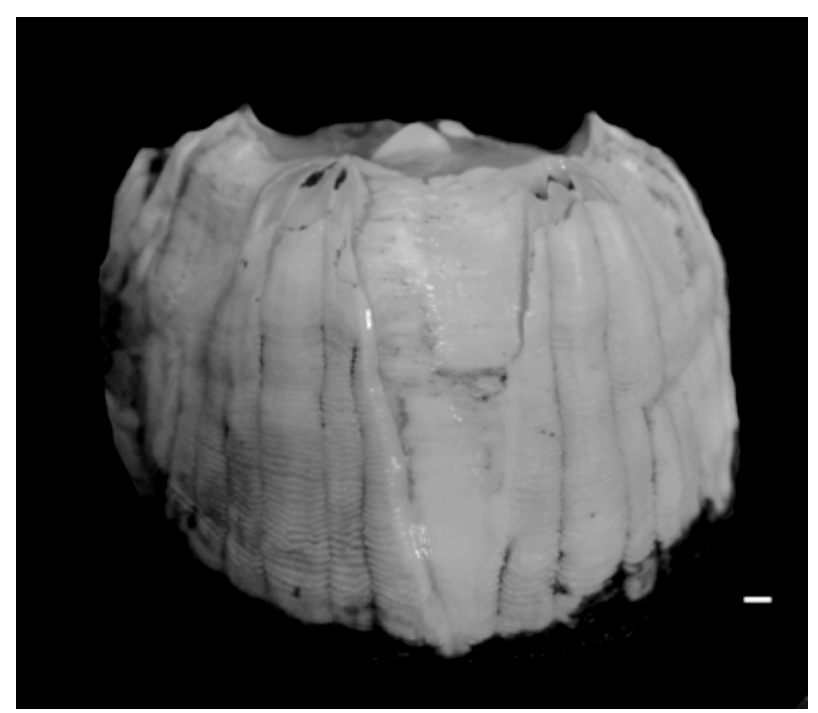

FIG. 2. Adulto de Coronula diadema recolectado en un ballenato de ballena jorobada en el PNN Gorgona. (Escala: $1 \mathrm{~mm}$ ).

Foto: Luis Miguel Cuellar.

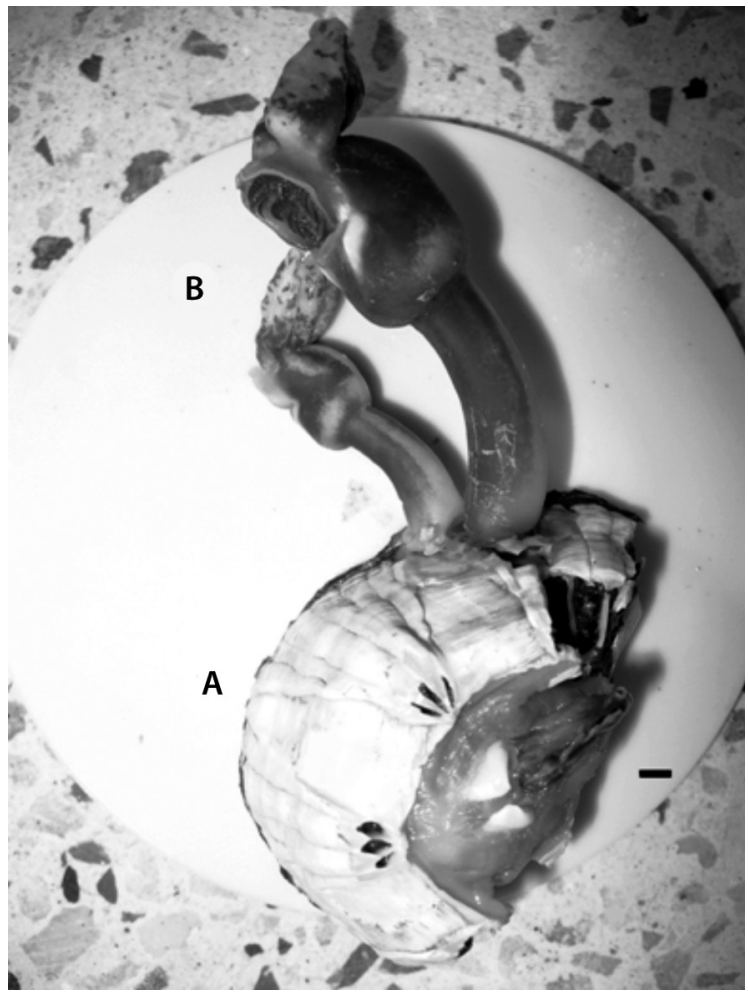

FIG. 3. Coronula diadema (A) con dos Conchoderma auritum (B) creciendo sobre él, recolectados de un ballenato de ballena jorobada en el PNN Gorgona. (Escala: 1mm).

Foto: Luis Miguel Cuellar.

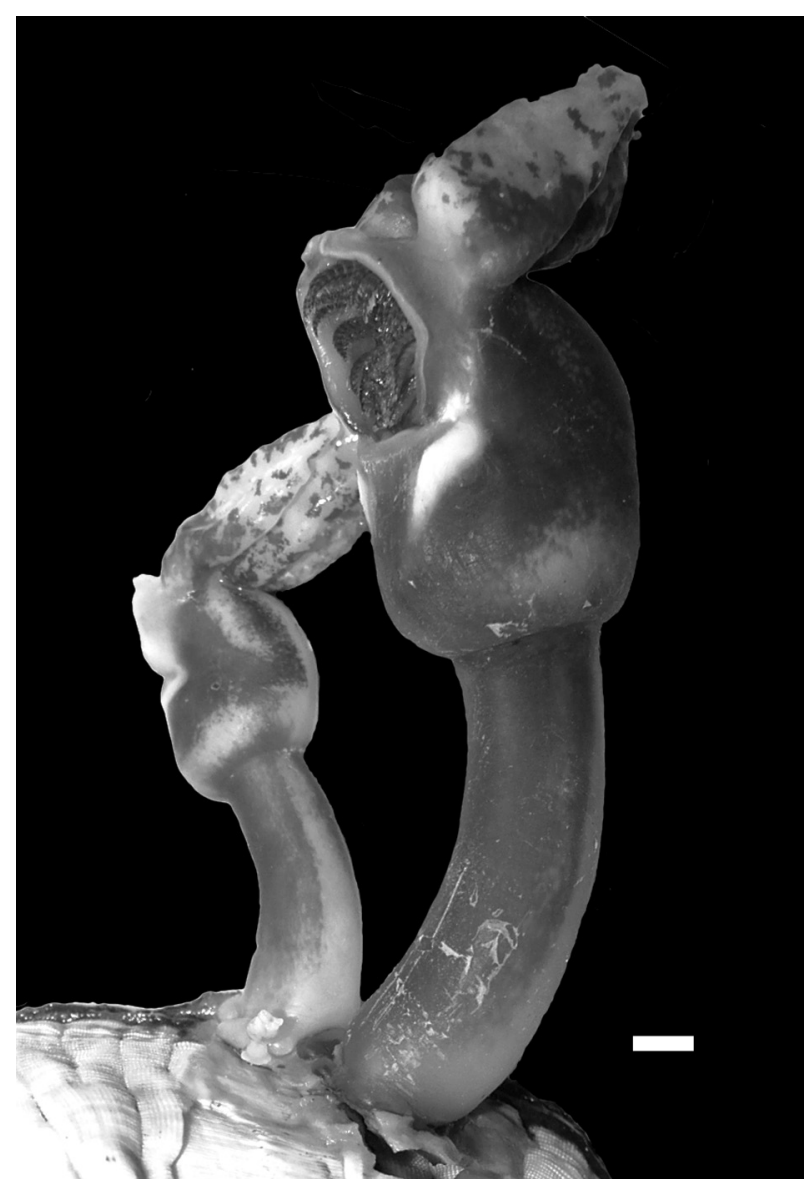

FIG. 4. Adulto de Conchoderma auritum recolectado de un ballenato de ballena jorobada en el PNN Gorgona.

(Escala: $1 \mathrm{~mm}$ ).

Foto: Luis Miguel Cuellar.

\section{DISCUSIÓN}

Tal como se ha reportado con otras especies de mamíferos marinos (e.g. Physeter macrocephalus, Hyperoodon ampullatus, Ziphius cavirostris, Berardius bairdii, Mesoplodon europaeus, M. bidens, Globicephala melas, Balaenoptera physalus, B. musculus y B. borealis) (Clarke 1966, Scarff 1986), la ballena jorobada sirve de huésped para diversos tipos de organismos ectoparásitos y epibiontes que se adhieren a su piel. La presencia de ectoprásitos y epibiontes en ballenas jorobadas ha sido registrada en altas y bajas latitudes (Cuadro 1). Cyamus boopis colectada en el ejemplar de Gorgona, en la base de la concha de C. diadema, ha sido registrada como ectoparásito especie específico para M. novaeangliae. Habitualmente, se encuentra adherido a la piel de la ballena jorobada cerca de su abertura genital (Scheffer 1939) y es conocido en algunas localidades como "piojo de ballenas" (Winn \& Reichley 1985). 


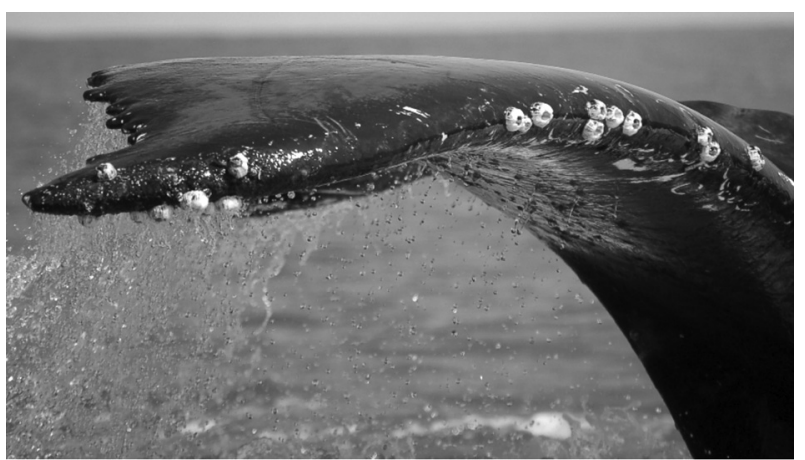

FIG. 5. Coronula diadema (aproximadamente $55 \mathrm{~mm}$ de diámetro) y Conchoderma auritum en el borde de la aleta caudal de un adulto de ballena jorobada en Bahía Málaga.

Foto: Isabel Cristina Avila.

Vea a color esta fotografía y las siguientes, en la versión digital (www.uned.ac.cr/investigacio/publicaciones/cuaderno1/).

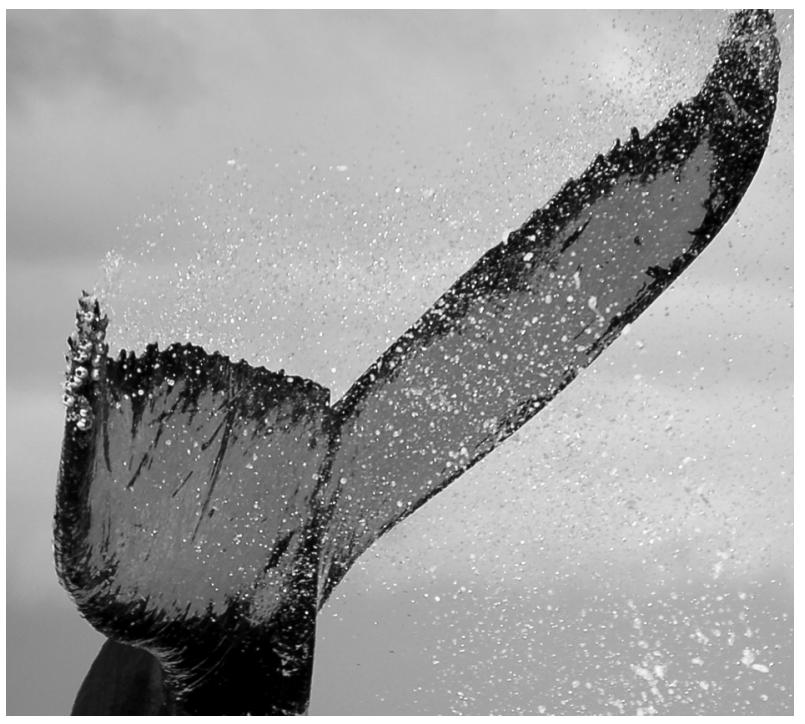

FIG. 6. Coronula diadema (aproximadamente $50 \mathrm{~mm}$ de diámetro) y Conchoderma auritum en el borde de la aleta caudal de un adulto de ballena jorobada en Bahía Málaga.

Foto: Isabel Cristina Avila.

En algunas localidades se conoce $C$. auritum como balano"orejas de conejo" o"flores de ballena"y es un comensal secundario de Coronula diadema (Scheffer 1939). Algunos autores mencionan que $C$. auritum no puede fijarse directamente sobre la piel de la ballena (Nilsson-Cantell 1930), y debido a esto, Conchoderma auritum necesita una superficie dura para su asentamiento, por lo que utiliza las conchas de Coronula, con una preferencia por C. diadema (Angot 1951). Esta preferencia se debe a que C. diadema

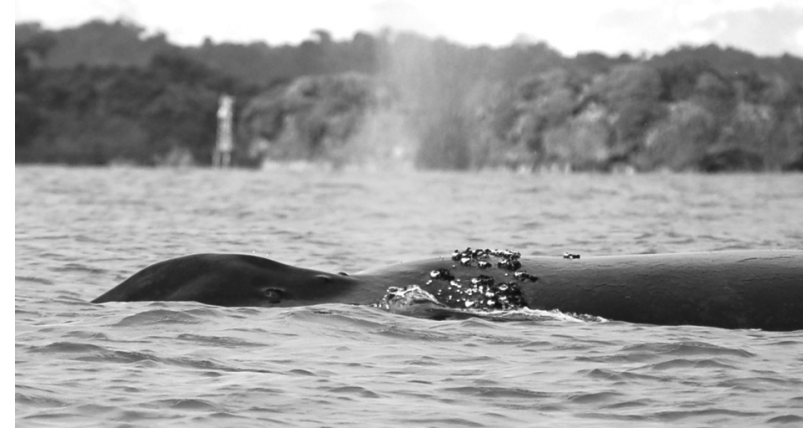

FIG. 7. Coronula diadema (aproximadamente $50 \mathrm{~mm}$ de diámetro) y Conchoderma auritum en el dorso, cerca al espiráculo, de un adulto de ballena jorobada en Bahía Málaga.

Foto: Isabel Cristina Avila.

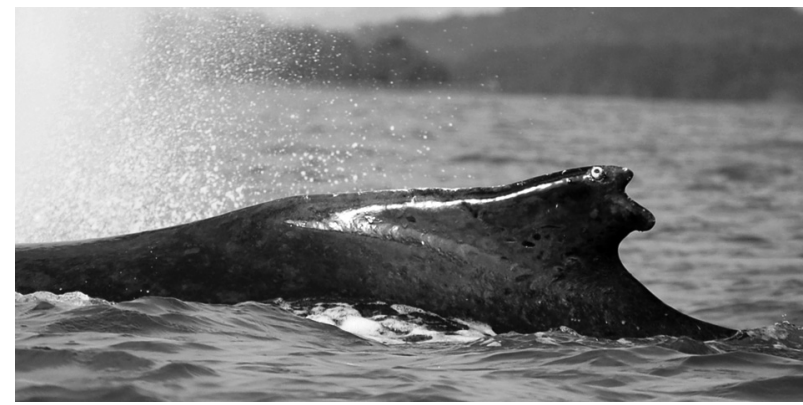

FIG. 8. Coronula diadema (aproximadamente $55 \mathrm{~mm}$ de diámetro) en la aleta dorsal de un adulto de ballena jorobada en Bahía Málaga.

Foto: Isabel Cristina Avila.

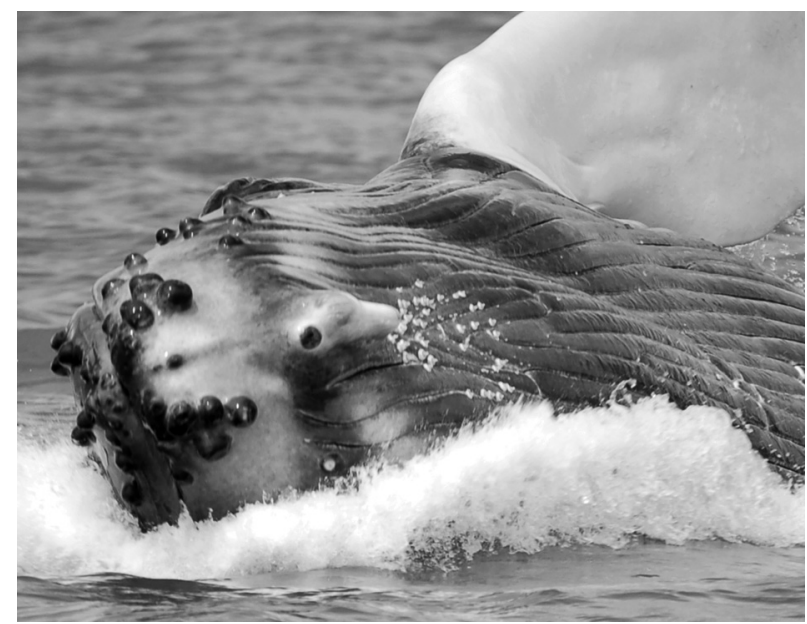

FIG. 9. Coronula diadema (aproximadamente $20 \mathrm{~mm}$ de diámetro) en la parte ventral de la mandíbula inferior de un ballenato de ballena jorobada en Bahía Málaga, nacido en la temporada 2010.

Foto: Isabel Cristina Avila. 
CUADRO 1

Fauna ectoparásita y epibionte reportada en ballenas jorobadas (Megaptera novaeangliae).

\begin{tabular}{|c|c|c|}
\hline $\begin{array}{l}\text { Especie de fauna ectoparásita } \\
\text { y epibionte }\end{array}$ & Región/localidad & Fuente \\
\hline \multirow[t]{11}{*}{ Conchoderma auritum } & Bahía Málaga, Colombia & Presente trabajo \\
\hline & PNN Gorgona, Colombia & Presente trabajo \\
\hline & Antártica & Clarke 1966 \\
\hline & Mar del Norte, Alemania & Holthuis \& Fransen 2004 \\
\hline & Islas Aleutian, USA & Scheffer 1939 \\
\hline & Islas Georgias del Sur & Clarke 1966 \\
\hline & Sur África & Clarke 1966 \\
\hline & Japón e Islas Bonin (Japón) & Clarke 1966 \\
\hline & Islas Ryukyu, Japón & Clarke 1966 \\
\hline & Puerto Rico & Cintron-De Jesus 2001 \\
\hline & Florida, USA & Cintron-De Jesus 2001 \\
\hline \multirow[t]{23}{*}{ Coronula diadema } & Puerto Rico & Cintron-De Jesus 2001 \\
\hline & Florida, USA & Cintron-De Jesus 2001 \\
\hline & Bahía Málaga, Colombia & Presente trabajo \\
\hline & PNN Gorgona, Colombia & Presente trabajo \\
\hline & Salinas, Ecuador & Félix et al. 2006 \\
\hline & Isla de Groenlandia & Clarke 1966 \\
\hline & Islandia & Clarke 1966 \\
\hline & Atlántico Norte & Scarff 1986, Clarke 1966 \\
\hline & Pacífico Norte & Scarff 1986, Clarke 1966 \\
\hline & Hemisferio Sur & Scarff 1986 \\
\hline & Estrecho de Bering & Clarke 1966 \\
\hline & Finnmark, Noruega & Clarke 1966 \\
\hline & Islas de South Shetlands, Antártica & Clarke 1966 \\
\hline & Nueva Zelanda & Clarke 1966 \\
\hline & British Columbia, Canadá & Clarke 1966 \\
\hline & Chikura, Japón & Nogata \& Matsumura 2006 \\
\hline & Islas Georgias del Sur & Clarke 1966 \\
\hline & Sur África & Clarke 1966 \\
\hline & Mar del Norte, Alemania & Holthuis \& Fransen 2004 \\
\hline & Islas Aleutian, USA & Clarke 1966 \\
\hline & Islas Aleutian, USA & Scheffer 1939 \\
\hline & California, USA & Clarke 1966 \\
\hline & Perú & Clarke 1966 \\
\hline \multirow[t]{4}{*}{ Coronula reginae } & Antártica & Clarke 1966 \\
\hline & California, USA & Clarke 1966 \\
\hline & Puerto Rico & Cintron-De Jesus 2001 \\
\hline & Todos los océanos & Scarff 1986 \\
\hline
\end{tabular}


CUADRO 1 (Continuación...)

Fauna ectoparásita y epibionte reportada en ballenas jorobadas (Megaptera novaeangliae).

\begin{tabular}{|c|c|c|}
\hline $\begin{array}{l}\text { Especie de fauna ectoparásita } \\
\text { y epibionte }\end{array}$ & Región/localidad & Fuente \\
\hline \multirow[t]{8}{*}{ Coronula sp. } & Islandia & Clarke 1966 \\
\hline & Pacífico Norte & Clarke 1966 \\
\hline & New England, USA & Clarke 1966 \\
\hline & Islas Georgias del Sur & Clarke 1966 \\
\hline & Sur África & Clarke 1966 \\
\hline & Madagascar & Clarke 1966 \\
\hline & Antártica & Clarke 1966 \\
\hline & Puerto Rico & Mignucci-Giannoni et al. 1998 \\
\hline \multirow[t]{7}{*}{ Cyamus boopis } & Bahía Málaga, Colombia & Presente trabajo \\
\hline & PNN Gorgona, Colombia & Presente trabajo \\
\hline & Islas Aleutian, USA & Scheffer 1939 \\
\hline & Mar del Norte, Alemania & Holthuis \& Fransen 2004 \\
\hline & Puerto Rico & Mignucci-Giannoni et al. 1998 \\
\hline & Truro, Massachusetts, USA & Rowntree 1996 \\
\hline & Pacífico canadiense, Canadá & Margolis 1954 \\
\hline Cyamus erraticus & Nueva Zelanda & Hurley 1952 \\
\hline Pollicipes polymerus & Estrecho de Bering & Clarke 1966 \\
\hline
\end{tabular}

tiene una forma de barril, lo que le ofrece una mayor superficie de asentamiento que otras especies de Coronula (Cornwall 1927). Aunque no se han catalogado como parásitos especificos de $M$. novaeangliae, este hospedero cuenta con la mayoría de los registros históricos (Clarke 1966). En un estudio realizado en el Caribe, se observó que $C$. auritum no es un epibionte especie-específico de M. novaeangliae (Mignucci-Giannoni et al. 1998).

Las ballenas jorobadas no son nadadoras rápidas. Su velocidad de desplazamiento oscila entre 1,6 y $4,2 \mathrm{~km} / \mathrm{h}$ aunque se ha registrado que podría alcanzar hasta $23,3 \mathrm{~km} / \mathrm{h}$ durante cortos periodos (Noad \& Cato 2007). La baja velocidad permite a los ectoparásitos y epibiontes adherirse a la piel de las ballenas y permanecer allí durante toda su vida. Sin embargo, la ballena jorobada parece tener mecanismos para deshacerse de estos crustáceos adheridos a su piel, los saltos de vientre, de cola y de giro, representan un comportamiento común en esta especie (Whitehead 1985; Avila 2000), y se considera que se asocia a la limpieza de parásitos adheridos a la piel (Tyack \& Whitehead 1983, Whitehead 1985, Félix et al. 2006).
Los ectoparásitos y los epibiontes registrados en este estudio pueden incidir en el desplazamiento de las ballenas, pues la presencia de ellos en las aletas caudal y dorsal, en la parte dorsal de la cabeza podría afectar su hidrodinámica. La presencia en masa de estos organismos, aumentaría el peso que el animal tiene que mover para desplazarse y moverse, aumentando entonces su consumo energético. Además, la presencia de epibiontes en la mandíbula inferior, en el caso del ballenato de Bahía Málaga, podría afectar la libre apertura de su boca, y por lo tanto afectaría su alimentación. Por otro lado, la presencia de ectoparásitos y epibiontes también podría afectar el comportamiento de las ballenas, aumentando la frecuencia de saltos, coletazos y aletazos, para poderse liberar de ellos.

El espécimen de Coronula diadema recolectado en el ballenato en el PNN Gorgona, se considera un adulto debido a su talla (Scarff 1986); además, ya que C. diadema vive a lo sumo un año (Cornwall 1955), se puede inferir que la edad del ballenato de Gorgona (que colisionó con la embarcación) era de aproximadamente un año. Además Nogata \& Matsumura (2006) encontraron que la larva de C. diadema tiene un crecimiento óptimo a $25^{\circ} \mathrm{C}$ y muere 
a temperaturas menores a $15^{\circ} \mathrm{C}$. Por lo anterior, es posible que los epibiontes se adhirieron al ballenato en aguas costeras tropicales, probablemente en Colombia (aguas con temperatura promedio de $25-26^{\circ} \mathrm{C}$, Cantera 1993). Por otra parte, el haber encontrado en Bahía Málaga un ballenato de a lo sumo tres meses de edad con epibiontes jóvenes, adheridos en la parte ventral de la mandíbula inferior, indica que el ballenato adquirió los epibiontes en Colombia, muy posiblemente en Bahía Málaga. Este resultado confirma que los balanos se pueden adherir a las ballenas en aguas tropicales, tal como lo afirman Clarke (1966), Slijper (1979) y Félix et al. (2006), y que además, estos epibiontes se establecen y permanecen tanto en ballenas adultas como en ballenatos de corta edad.

\section{AGRADECIMIENTOS}

A los funcionarios de Parques Nacionales residentes en Isla Gorgona, especialmente a Ximena Zorrilla, Javier Solís, Elizabeth Hernández, Héctor Chirimia, Luis Payán, Jesús Agüiño, Justin y Hercilio por su apoyo en la recolección y análisis de los ectoparásitos y organismos desprendidos. A Alberto Parra, Francisco Javier Álvarez, Marie Van Bressem, Anais Van Bressem y Elías Álvarez por su apoyo en el trabajo de campo en Bahía Málaga y en la consecución de las fotografías. A Koen Van Waerebeek por el apoyo en la confirmación de la identificación de los ectoparásitos y epibiontes.

\section{REFERENCIAS}

Acevedo J, K. Rasmussen, F. Félix, C. Castro, M. Llano, E.R Secchi, M.T Saborío, A. Aguayo Lobo, B. Haase, M. Scheidat, L. DaIla-Rosa, C. Olavarría, P. Forestell, P. Acuña, G. Kaufman \& L. Pastene. 2007. Migratory destinations of the humpback whales from Magellan Strait feeding ground, Chile. Marine Mammal Science 23: 453-463.

Angot, M. 1951. Rapport scientifique sur les expéditions balenihes autour de Madagascar (saisons 1949 et 1950). Mémoires de I'Institut Scientifique de Madagascar 2: 439-486.

Avila, I.C. 2000. Algunos aspectos en el comportamiento superficial de la ballena jorobada (Megaptera novaeangliae) en los diferentes grupos conformados alrededor del par madre-cría en el Pacífico colombiano. Tesis de pregrado. Universidad del Valle, Facultad de Ciencias, Programa académico de Biología. Santiago de Cali, Colombia.

Balbuena J.A., F.J. Aznar, M. Fernandez \& J.A. Raga. 1995. Parasites as indicators of social structure and stock identity of marine mammals. Developments in Marine Biology 4: 133-139.
Bianucci, G., W. Landini \& J.S. Buckeridge. 2006. Whale barnacles and Neogene cetacean migration routes. New Zealand Journal of Geology and Geophysics 49: 115-120.

Capella, J., L. Flórez-González \& P. Falk. 2001. Mortality and anthropogenic harassment of humpback whales along the Pacific coast of Colombia. Memoirs of the Queensland Museum 47: 547-553.

Cantera, J. 1993. Oceanografía, p. 12-23. In: Pablo Leiva (ed.). Colombia Pacífico. Tomo I. Fondo FEN Colombia, Santa Fé de Bogotá, Colombia.

Cintron-De Jesus, J. 2001. Barnacles associated with marine vertebrate in Puerto Rico and Florida. Tesis de Maestría, Facultad de Artes y Ciencias, Mayagûez-Puerto Rico. University of Puerto Rico, Mayagüez, Puerto Rico.

Clapham, P. J. 2000. The humpback whale: seasonal feeding and breeding in a baleen whale, p. 173-196. In: J. Mann, R. C. Connor, P. Tyack \& H. Whitehead (eds.). Cetacean Societies: Field Studies of dolphin and whales. The University of Chicago, Chicago, EEUU.

Clarke, R. 1966. The stalked barnacle Conchoderma, ectoparasitic on whales. Norsk Hvalfangst-Tidende 55: 153-168.

Corkeron, P.J. 1995. Humpback whales. (Megaptera novaeangliae) in Hervey Bay, Queensland: behaviour and responses to whale-watching vessels. Canadian Journal of Zoology 73: 1290-1299.

Cornwall, I.E. 1927. Some North Pacific whale barnacles. Contributions to Canadian Biology and Fisheries 3: 503-517.

Cornwall, I. E. 1955. The barnacles of British Columbia. British Columbia Provincial Museum Department of Education Handbook 7: 5-69.

Dawbin, W.H. 1966. The seasonal migratory cycle of humpback whales, p. 145-170. In: Kenneth S. Norris (ed.). Whales, dolphins and porpoises. University of California, California, EEUU.

Dyer, B.D. 1989. Symbiosis and Organismal Bondaries. American Zoologist 29: 1085-1093.

D'Vincent, C., D. Haley \& F.A. Sharpe. 1989. Voyaging with the whales. Boulton Publishing, Ontario, Canada.

Félix, F., B. Bearson, \& J. Falconí. 2006. Epizoic barnacles removed from the skin of a humpback whale after a period of intense surface activity. Marine Mammal Science 22: 979-984.

Fertl, D. \& W.A. Newman. 2009. Barnacles, p: 75-78. In: W.F. Perrin, B. Würsig \& J.G.M. Thewissen (eds.). Encyclopedia of Marine Mammals. Second edition. Academic, California, EEUU.

Flórez-González, L., J.J. Capella \& H.C. Rosenbaum. 1994. Attack of killer whales (Orcinus orca) on humpback whales (Megaptera novaeangliae) on a South American Pacific breeding ground. Marine Mammal Science 10: 218-222.

Ford, J. \& R.R. Reeves. 2008. Fight or flight: antipredator strategies of baleen whales. Mammal Review 38: 50-86. 
Holthuis, L.B. \& C.H.J.M. Fransen. 2004. Interesting records of whale epizoic crustaceans from the Dutch North Sea coast (Cirripedia, Amphipoda). Nederlandse Faunistische Mededelingen 21: 11-16.

Hurley, D.E. 1952. Studies on the New Zealand amphipodan fauna No. 1-The family Cyamidae: the whale-louse Paracyamus boopis. Transactions of the Royal Society of New Zealand 80: 63-68.

Laist, D.W., A.R. Knowlton, J.G. Mead, A.S. Collet \& M. Podesta. 2001. Collisions between ships and whales. Marine Mammal Science 17: 35-75.

Lambertsen, R.H. 1986. Disease of the common fin whale (Balaenoptera physalus): Crassicaudiosis of the urinary system. Journal of Mammalogy 67: 353-366.

Mackenzie, K. 2002. Parasites as biological tags in population studies of marine organisms: an update. Parasitology 124: 153-163.

Margolis, L. 1954. Three kinds of whale-lice (Cyamidae: Amphipoda) from the Pacific coast of Canada, including a new species Journals of the Fisheries Research Board Canada 11: 319-325.

Mignucci-Giannoni A.A., E.P. Hoberg, D. Siegel-Causey \& E.H. WiIliams. 1998. Metazoan parasites and other symbionts of cetaceans in the Caribbean. The Journal of Parasitology 84: 939-946.

Nilsson-Cantell C.A. 1930. Thoracic cirripedes collected in 19251927. Discovery Reports 2: 223-260.

Noad, M.J. \& D.H. Cato. 2007. Swimming speeds of singing and non-singing humpback whales during migration. Marine Mammal Science 23: 481-495

Nogata Y. \& K. Matsumura. 2006. Larval development and settlement of a whale barnacle. Biology Letters 2: 92-93.

Perrin, W.F. 1969. The Barnacle, Conchoderma auritum, on a Porpoise (Stenella graffmani). Journal of Mammalogy 50: 149-151.

Pierotti, R., C.A. Swatland \& P.W. Ewald. 1985. "Brass knuckles" in the sea: the use of barnacles as weapons, p: 60. In: Proceedings of the Sixth Biennial Conference on the Biology of Marine Mammals. Marine Mammalogy, Vancouver, Columbia Británica, Canadá.

Raga, J.A., M. Fernández, J.A. Balbuena \& F.J. Aznar. 2009. Parasites, p: 867-876. In: W.F. Perrin, B. Würsig \& J.G.M. Thewissen (eds.). Encyclopedia of Marine Mammals, Second edition. Academic, California, EEUU.

Rowntree, V.J. 1996. Feeding, distribution, and reproductive behavior of cyamids (Crustacea: Amphipoda) living on humpback and right whales. Canadian Journal of Zoology 74: 103-109.
Scarff, J.E. 1986. Occurrence of the barnacles Coronula diadema, C. reginae and Cetopirus complanatus (Cirripedia) on right whales. The Scientific Reports of the Whales Research Institute 37: 129-153.

Scheffer. V. 1939. Organisms collected from whales in the Aleutian Islands. Murrelet 20: 67-69.

Slijper, E.J. 1962. Whales: the biology of cetaceans. Basic Books, New York, EEUU.

Slijper, E.J. 1979. Whales. Cornell University, New York, EEUU.

Stevick, P., A. Aguayo, J. Allen, I.C. Avila, J. Capella, C. Castro, K. Chater, L. Dalla-Rosa, M.H. Engel, F. Félix, L. Flórez-González, A. Freitas, B. Haase, M. Llano, L. Lodi, E. Muñoz, C. Olavarría, E. Secchi, M. Scheidat \& S. Siciliano. 2004. A note on the migrations of individually identified humpback whales between the Antarctic Peninsula and South America. Journal of Cetacean Research and Management 6: 109-113.

Tyack, P. \& H. P. Whitehead. 1983. Male competition in large groups of wintering humpback whales (Megaptera novaeangliae). Behavior 83: 132-154.

Van Bressem M-F, J.A. Raga, G. Di Guardo, P.D. Jepson, P. Duignan, U. Siebert, T. Barrett, M.C.O. Santos, I.B. Moreno, S. Siciliano, A. Aguilar \& K. Van Waerebeek. 2009. Emerging infectious diseases in cetaceans worldwide and the role of environmental stressors. Diseases of Aquatic Organisms 86: 143-157.

Van Waerebeek, K., A.N. Baker, F. Félix, J. Gedamke, M. Iñiguez, G.P. Sanino, E. Secchi, D. Sutaria, A. Van Helden \& Y. Wang. 2007. Vessel collisions with small cetaceans worldwide and with large whales in the Southern Hemisphere, an initial assessment. Latin American Journal of Aquatic Mammals 6: 43-69.

Yuk-Maan, L., 1967. An illustrated key to the species of whale-lice (Amphipoda, Cyamidae), ectoparasites of cetacea, with a guide to the literature. Crustaceana 12: 279-291.

Weller, D.W. 2002. Predation on marine mammals, p: 985-994. In: W.F. Perrin, B. Würsig \& J.G.M. Thewissen (eds.). Encyclopedia of Marine Mammals, Academic, California, EEUU.

Whitehead, H. 1985. Humpback whale breaching. Investigations on Cetacea 17:117-155.

Whitehead, H.P. \& C. Glass. 1985. Orcas (killer whales) attack humpback whales. Journal of Mammalogy 66: 183-185.

Winn, H.E. \& N.E. Reichley. 1985. Humpback whale, Megaptera novaeangliae, p: 241-273. In: S.H. Ridgway \& R. Harrison (eds.). Handbook of Marine Mammals Vol. 3: the sirenians and baleen whales. Academic, Londres, United Kingdom. 
